EPJ Web of Conferences 40, 12002 (2013)

DOI: $10.1051 /$ epjconf/20134012002

(C) Owned by the authors, published by EDP Sciences, 2013

\title{
Scaling of Hall coefficient in Co-Bi granular thin films
}

\author{
P. Athanasopoulos ${ }^{1}$, C. Christides ${ }^{1}$, and Th. Speliotis ${ }^{2}$ \\ ${ }^{1}$ School of Engineering, University of Patras, 26504 Rion Patras, Greece \\ ${ }^{2}$ Institute of Materials Science, NCSR Demokritos, 15310 Aghia Paraskevi, Athens, Greece
}

\begin{abstract}
A series of Co-Bi thin films with Co concentrations $\mathrm{c}=0,0.05,0.2,0.26,0.3,0.333,0.375,0.545$, were grown by magnetron sputtering on $\mathrm{Si}(100) / \mathrm{SiN}_{\mathrm{X}}$ substrates. Resistivity measurements at zero field $\left(\rho_{\mathrm{xx}}\right)$ as a function of temperature- $T$ exhibit an exponential variation with $\mathrm{T}$ in the region of $240 \mathrm{~K}<\mathrm{T}<300 \mathrm{~K}$. The Hall coefficient as a function of Co concentration diverges as $\log |\mathrm{c}-0.3|^{0.3}$ for $\mathrm{c}<0.333$, indicating a scaling of $\mathrm{R}_{\mathrm{H}}$ nearby a percolation threshold $\mathrm{p}_{\mathrm{c}}=0.3$. Only after proper scaling of the anomalous Hall coefficient $\mathrm{R}_{\mathrm{S}}$ the conventional $\mathrm{R}_{\mathrm{S}} \propto\left(\rho_{\mathrm{xx}}\right)^{\mathrm{n}}$ dependence can be satisfied.
\end{abstract}

\section{Introduction}

In magnetic films the Hall resistivity $\rho_{\mathrm{H}}$ is defined by the phenomenological equation:

$\rho_{H}=R_{0} B+R_{S} \mu_{o} M$

where $\mathbf{B}$ is the magnetic induction perpendicular to film surface, $\mathrm{R}_{0}$ is the ordinary Hall $(\mathrm{OH})$ coefficient, $\mathbf{M}$ is the magnetization, and $R_{S}$ is the anomalous Hall (AH) coefficient. Usually, in ferromagnetic metals (FM), $R_{S}$ satisfies a scaling law [1] with the longitudinal film resistivity $\rho_{\mathrm{xx}}$ measured at zero applied magnetic field:

$R_{S}=a \rho_{x x}+b \rho_{x x}^{2}$

where the first term is assigned to skew scattering and the second term to side jump mechanism [1]. Generally, three regimes were observed [1] with respect to the dependence of anomalous Hall conductivity (AHC) on the conductivity $\sigma_{\mathrm{xx}}$. Equation-2 is valid within the metallic regime [1] whereas in the insulating regime other scaling exponents were observed [2]. Bismuth films may exhibit p-type or n-type conduction properties, depending on film texture [3] and the participation of surface states on the different crystallographic faces [4]. In our study Cobalt (FM) and semimetal Bismuth layered thin film structures are used to investigate the effect of $\mathrm{FM} /$ semimetal junctions in Hall resistivity measurements.

\section{Experimental Details}

A series of thin film $\mathrm{Co} / \mathrm{Bi}$ structures were grown by magnetron sputtering on $\mathrm{Si}(100) / \mathrm{SiN}_{\mathrm{X}}$ substrates with rectangular shapes of $5 \times 5 \mathrm{~mm}^{2}$, using a base pressure of Ar-gas about $10^{-8}$ Torr, without to control the substrate temperature during film deposition. Specifically, the sample compositions are: two pure Bi films with $15 \mathrm{~nm}$ and $50 \mathrm{~nm}$ thicknesses, denoted as $\mathrm{Bi}(15 \mathrm{~nm})$ and $\mathrm{Bi}(50 \mathrm{~nm})$, two trilayer structures of $\mathrm{Bi}(10 \mathrm{~nm}) / \mathrm{Co}(1 \mathrm{~nm}) /$ $\mathrm{Bi}(10 \mathrm{~nm})$, and $\mathrm{Bi}(10 \mathrm{~nm}) / \mathrm{Co}(5 \mathrm{~nm}) / \mathrm{Bi}(10 \mathrm{~nm})$, one bilayer structure of $\mathrm{Bi}(10 \mathrm{~nm}) / \mathrm{Co}(5 \mathrm{~nm})$, and four multilayer structures with each one composed by ten repetitions of the same $[\mathrm{Bi} / \mathrm{Co}]$ bilayer unit, denoted as $[\mathrm{Bi}(0.7 \mathrm{~nm}) /$ $\mathrm{Co}(2 \mathrm{~nm})]_{10}, \quad[\mathrm{Bi}(1 \mathrm{~nm}) / \mathrm{Co}(2.4 \mathrm{~nm})]_{10}, \quad[\mathrm{Bi}(2.4 \mathrm{~nm}) /$ $\mathrm{Co}(4 \mathrm{~nm})]_{10}$, and $[\mathrm{Bi}(2.4 \mathrm{~nm}) / \mathrm{Co}(4 \mathrm{~nm})]_{10}$. The nominal thickness values of $\mathrm{Co}$ and $\mathrm{Bi}$ layers were reduced to $\mathrm{Co}$ concentrations, c-Co, $(0,0.05,0.2,0.26,0.3,0.333$, $0.375,0.545)$ by dividing the subtotal of Co layer thickness with total film thickness. The results in Fig.2 are tabulated with increasing order of c-Co.

Resistivity and magneto-transport measurements were performed on the as-made films with a PPMS, using the Van der Pauw method in magnetic fields up to $B=9 T$ perpendicular to film-plane. All raw data collected from Hall resistance measurements were corrected according to Ref.[5] to obtain the final loops of $\rho_{H}$ vs B and Hall coefficient $R_{H}$ vs $B$ shown in Figs.1, 2. X-ray diffraction (XRD) measurements reveal that all films are polycrystalline. Specifically, the predominant Braggpeaks with $(00 l)$ indices $(l=3,6)$, observed in pure Bi films, decrease towards to zero intensity as c increases, indicating a progressive change in texture of $\mathrm{Bi}$ layers with c-Co.

\section{Results}

Fig.1a shows representative $\rho_{\mathrm{H}}$ vs $\mathrm{B}$ data and isothermal magnetization $\mathrm{M}$ vs $\mathrm{B}$ loops observed in Co-rich film at $5 \mathrm{~K}$. Fig. $1 \mathrm{~b}$ shows three selected $\mathrm{M}$ vs $\mathrm{B}$ loops at $5 \mathrm{~K}$ that span the c-Co range, indicating that the saturation magnetization $\mathrm{M}_{\mathrm{S}}$ values are strongly affected by c-Co

This is an Open Access article distributed under the terms of the Creative Commons Attribution License 2.0, which permits unrestricted use, distribution, and reproduction in any medium, provided the original work is properly cited. 


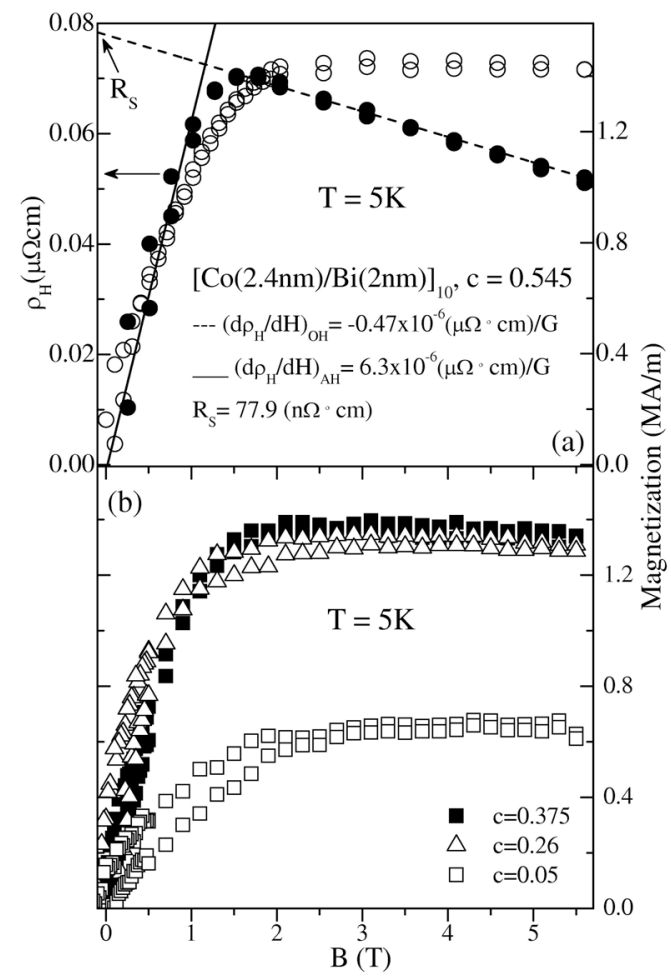

Fig.1. (a) Indicative Hall resistivity $\rho_{H}(\bullet)$ and magnetization-M (o) loops that explain the estimation of $\mathrm{R}_{0}$ from the high-field slope and $R_{\mathrm{S}}$ from the point of intersection with $\rho_{\mathrm{H}}$-axis. (b) Selected isothermal magnetization loops, showing that saturation of magnetization $\mathrm{M}_{\mathrm{S}}$ occurs at $\mathrm{B}_{\mathrm{S}} \approx 2 \mathrm{~T}$ for $\mathrm{T}=5 \mathrm{~K}$.

whereas the saturation field $\mathrm{B}_{\mathrm{S}}$ (and thus magnetic anisotropy) are not influenced by $\mathrm{c}-\mathrm{Co}$ at a fixed temperature. Thus, the non linear dependence of the Hall coefficient $R_{H}=\rho_{H} / B$ on $B$, observed in Fig.2, cannot be attributed to magnetization effects above $2 \mathrm{~T}$. In addition, Fig.2a shows that pure Bi film exhibits non linear $R_{H}$ vs $\mathrm{B}$ curves above 2 Tesla for $\mathrm{T} \leq 100 \mathrm{~K}$. As we show in a forthcoming publication, these results cannot be fitted with equations used in the two-band model. The observed $\mathrm{R}_{\mathrm{H}}$ vs $\mathrm{B}$ curves in Fig. 2 can be explained by considering the galvanomagnetic properties of polycrystalline or inhomogeneous metals that depend strongly on the shape and the orientation of the crystallites relative to magnetic field direction [6]. The most important effect of crystalline shape and orientation in these films is the observed change of polarity in $R_{H}$ vs $B$ loops, from positive to negative in Fig.2, that does not depend on a systematic way from c-Co. As shown in Fig.3, the sign of $R_{H}(8 T)$, which is the value of $R_{H}$ at $8 T$ in Fig.2, and the sign of $R_{S}$, both change from negative to positive as the percentage of preferable orientation of [00l]-directions in $\mathrm{Bi}$ layers change from perpendicular to parallel in film plane. Note that both, the $R_{0}=d \rho_{H} / d H$ and the $R_{H}(8 T)$ values have positive sign in pure $\mathrm{Bi}$ films (Fig.3a), indicating that holes are the majority charge carriers [7]. In addition, Fig.3a reveals that $\mathrm{R}_{\mathrm{H}}(8 \mathrm{~T})$ becomes negative in between $0.2<c<0.375$, indicating a transition from $\mathrm{p}$ type (holes) to n-type (electrons) majority charge carriers in this region. This transition in $\mathrm{R}_{\mathrm{H}}(8 \mathrm{~T})$ values with c-Co is plotted together with $\mathrm{R}_{0}$ vs c-Co in Fig.4.

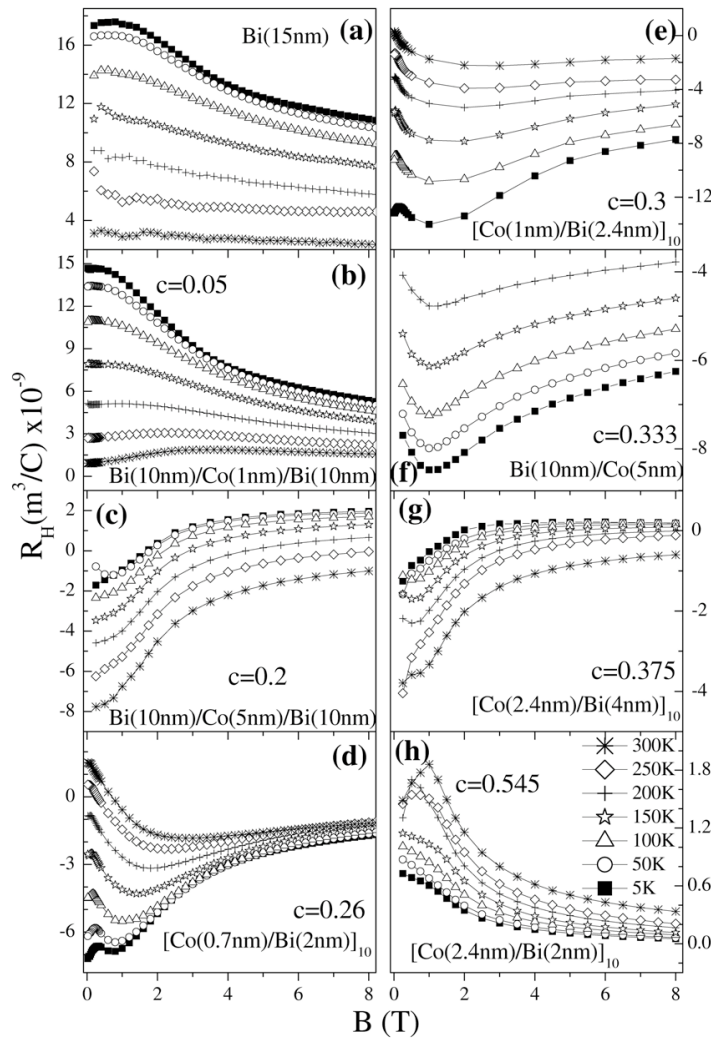

Fig. 2. Hall coefficient $R_{H}$ vs $B$ curves between $5 \mathrm{~K}$ and $300 \mathrm{~K}$.
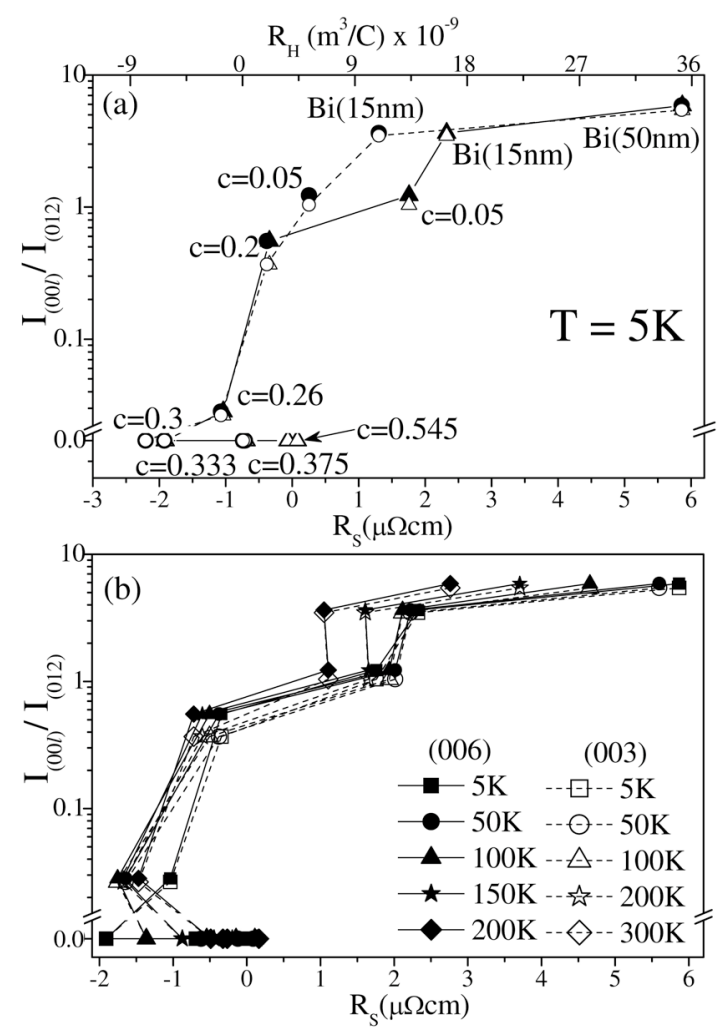

Fig. 3. Semi-log plot of XRD intensities from Bi (00l) Bragg peaks $(l=3$ or $l=6)$ as a function of: (a) $\mathrm{R}_{\mathrm{H}}(8 \mathrm{~T})$ values (dash line) and $R_{S}$ values (solid line) at $5 \mathrm{~K}$, and (b) $R_{S}$ values in the range of $5 \mathrm{~K} \leq \mathrm{T} \leq 200 \mathrm{~K}$. The $(003)$ and $(006)$ indices are plotted with open and dark symbols, respectively. Their intensities were normalized to observed (012) Bragg-peak intensity of Bi. 


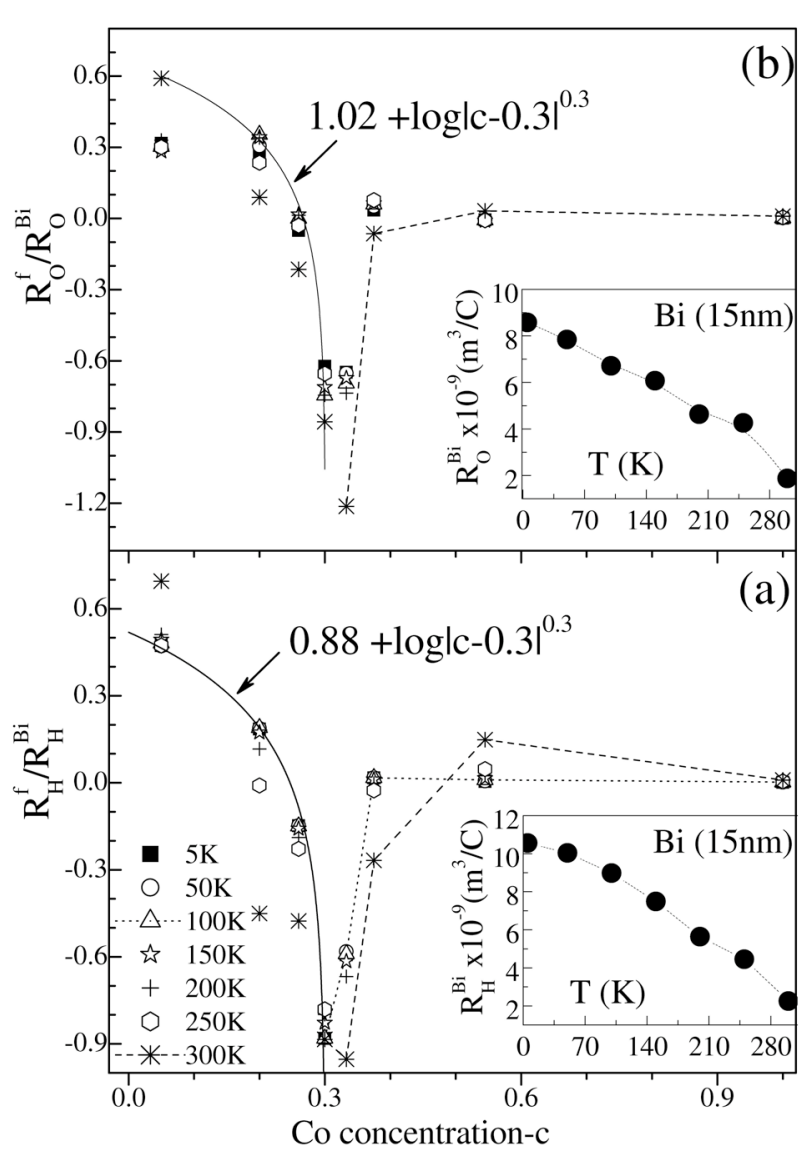

Fig. 4. Scaling of (a) $R_{H}^{f}$ and (b) $\mathrm{R}_{0}$ values with c-Co. Solid lines are fitting curves to equation shown with arrow. Insets show the temperature dependence of $R_{H}^{B i}$ and $R_{0}^{B i}$ values.

A plot of $R_{0}$ values instead of $R_{H}(8 T)$ in Fig.3a will exhibit the same trend, but we plot only the $R_{H}(8 T)$ values for reasons explained below. Fig.3b shows that the sign of $R_{S}$ does not follow the systematic sign reversal of $R_{H}(8 T)$ or $R_{0}$ with c-Co, but depends only on the degree of orientation of $[00 l]$-directions in Bi layers relative to film vertical direction. Since Bi exhibits [4] a strong spinorbit (SO) level splitting along [00l]-directions, then Fig.3b may imply that the SO Hamiltonian [8] $\mathrm{H}_{\mathrm{SO}}$ in $\mathrm{Bi}$ is negative for example when $[00 l]$-directions are inplane and positive for out-of-plane, thus, reversing from "left to right" direction the effective nonzero transverse electric field created by the SO interaction. In this case a junction with Co may give rise to unequal occupancy of spin-up and spin down states in $\mathrm{Co} / \mathrm{Bi}$ interfaces. This may contribute a term of interspin band scattering [8] to $\mathrm{AH}$ effect (AHE), in addition to intrinsic SO coupling term that contributes to AHE with the scattering of itinerant carriers due either to phonons or to thermal spin disorder.

Fig.4a shows normalized Hall coefficient $R_{H}^{f}$ values (obtained from Fig.2 at $\mathrm{B}=8 \mathrm{~T}$ for every film) to corresponding $\mathrm{Bi}$ film values $R_{H}^{B i}$ (see the inset) as a function of c-Co. The notation is that superscripts $f$ and $B i$ on $\mathrm{R}_{\mathrm{H}}, \mathrm{R}_{\mathrm{S}}$ and $\rho_{\mathrm{xx}}$ parameters indicate that each one is measured on a film of given $\mathrm{c}-\mathrm{Co} \neq 0$ and on pure $\mathrm{Bi}$ film

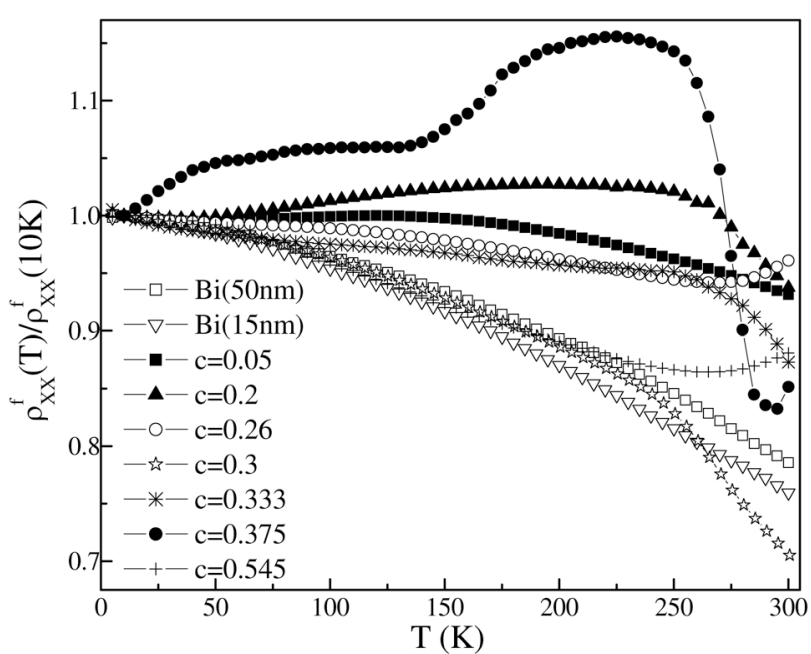

Fig. 5. Temperature dependence of the longitudinal film resistivity $\rho_{\mathrm{xx}}$ observed at zero external field in films with different Co concentrations. These $\rho_{\mathrm{xx}}(\mathrm{T})$ curves are normalized to the corresponding film $\rho_{\mathrm{xx}}$ value observed at $\mathrm{T}=10 \mathrm{~K}$. Lines are guides to the eye.

(c-Co=0), respectively. The $R_{H}^{f}$ values were found to diverge as $\log |\mathrm{c}-0.3|^{0.3}$ for $\mathrm{c}<0.333$, evidencing a critical behaviour [9] nearby a percolation threshold $\mathrm{p}_{\mathrm{c}}=0.3$, with critical exponent $g=0.3$. The physical origin of $\mathrm{p}_{\mathrm{c}}=0.3$ might be related to energy balance between the work functions [10] of Co and Bi layers and their Fermi level matching at $\mathrm{Co} / \mathrm{Bi}$ interfaces, that may create [11] a depletion layer (defining a critical length scale) of holelike or electron-like carriers in $\mathrm{Bi}$ as a function of c-Co. In addition, the same trend of $R_{H}^{f}$ vs c-Co appears in all $R_{H}^{f}(B)$ values determined for $\mathrm{B} \geq 5 \mathrm{~T}$. However, a plot of the ordinary Hall coefficient $\mathrm{R}_{0}$ with c-Co in Fig. $4 \mathrm{~b}$ (determined by the high-field slope in Fig.2, with $\mathrm{B}>3 \mathrm{~T}$ ) reveals a slightly shifted $\mathrm{p}_{\mathrm{c}}$ value at a value of $\mathrm{c}-\mathrm{Co} \approx$ 0.333. A comparison of c-Co values in Figs.3, 4 and 5 (see the inset), where a minimum of $I(00 l), R_{H}^{f}$ or $\mathrm{R}_{0}$, and $R_{S}$ values occurs, indicates that the $p_{c}$ value is more likely to be nearby $\mathrm{c}-\mathrm{Co}=0.3$. In addition, the same function, $\log |\mathrm{c}-0.3|^{0.3}$, fits (solid line) both data sets in Figs. $4 \mathrm{a}$ and $4 \mathrm{~b}$ for $\mathrm{c}-\mathrm{Co} \leq 0.3$ in the region of $5 \mathrm{~K} \leq \mathrm{T} \leq 250 \mathrm{~K}$.

Fig.5 shows the temperature dependence of $\rho_{x x}$ observed in all films at zero applied field. Remarkably, all these films exhibit an exponential variation of $\rho_{\mathrm{xx}}(\mathrm{T})$ at $240 \mathrm{~K}<\mathrm{T}<300 \mathrm{~K}$ whereas in some films $(\mathrm{c}=0.05,0.2$, $0.375)$ appears a $\rho_{x x} \approx T^{n}$ behavior for $T<240 K$. The exponential dependence in $\rho_{\mathrm{xx}}(\mathrm{T})$ curves arises from the dominant contribution of $\mathrm{Bi}$ layers to film resistivity, since the observed $\rho_{x x}^{B i}(T)$ curves of pure Bi films can be simulated with a single exponential function of $\mathrm{T}$ in the region of $5 \mathrm{~K} \leq \mathrm{T} \leq 300 \mathrm{~K}$.

The dominant contribution of $\rho_{x x}^{B i}(T)$ in the magnetotransport properties of our films can be clearly demonstrated if we try to satisfy Eq.2 for every c-Co by plotting the $\mathrm{R}_{\mathrm{S}}(\mathrm{T}) / \rho_{\mathrm{xx}}(\mathrm{T})$ ratio against the corresponding values of $\rho_{\mathrm{xx}}(\mathrm{T})$ from Fig.5. Fig.6 includes two such plots where all parameters are normalized to their values at $5 \mathrm{~K}$ 
in order to be able to compare the results for all c-Co values. Fig.6a shows that Eq. 2 is not satisfied when the measured value $\rho_{x x}^{f}(T)$ of the corresponding film (in Fig.5) is used, and the inset demonstrates that $R_{S}$ values as a function of $\mathrm{Co}$ concentration at $5 \mathrm{~K}$ exhibit a minimum at the same $\mathrm{p}_{\mathrm{c}}=0.3$ as the $R_{H}^{f}$ in Fig. $4 \mathrm{a}$. However, when we keep the actual $\mathrm{R}_{\mathrm{S}}(\mathrm{T})$ values for each c-Co and replace the $\rho_{x x}^{f}(T)$ values with the corresponding $\rho_{x x}^{B i}(T)$ values observed in $\operatorname{Bi}(15 \mathrm{~nm})$ film (from Fig.5) then Fig.6b shows that Eq.2 is satisfied for all the films.

The reason that Eq.2 is satisfied only with $\rho_{x x}^{B i}(T)$ but is not satisfied with $\rho_{x x}^{f}(T)$, is not clear. Preliminary results from analysis of the experimental data shown in Figs.5 and 6 provide evidence that the conventional scaling of $\mathrm{R}_{\mathrm{S}}$ in Eq.2 is maintained by substituting $\rho_{x x}^{B i}(T)$ because of a proper scaling [12] that involves a critical length scale in $\mathrm{Bi}$ side of $\mathrm{Bi} / \mathrm{Co}$ junctions.
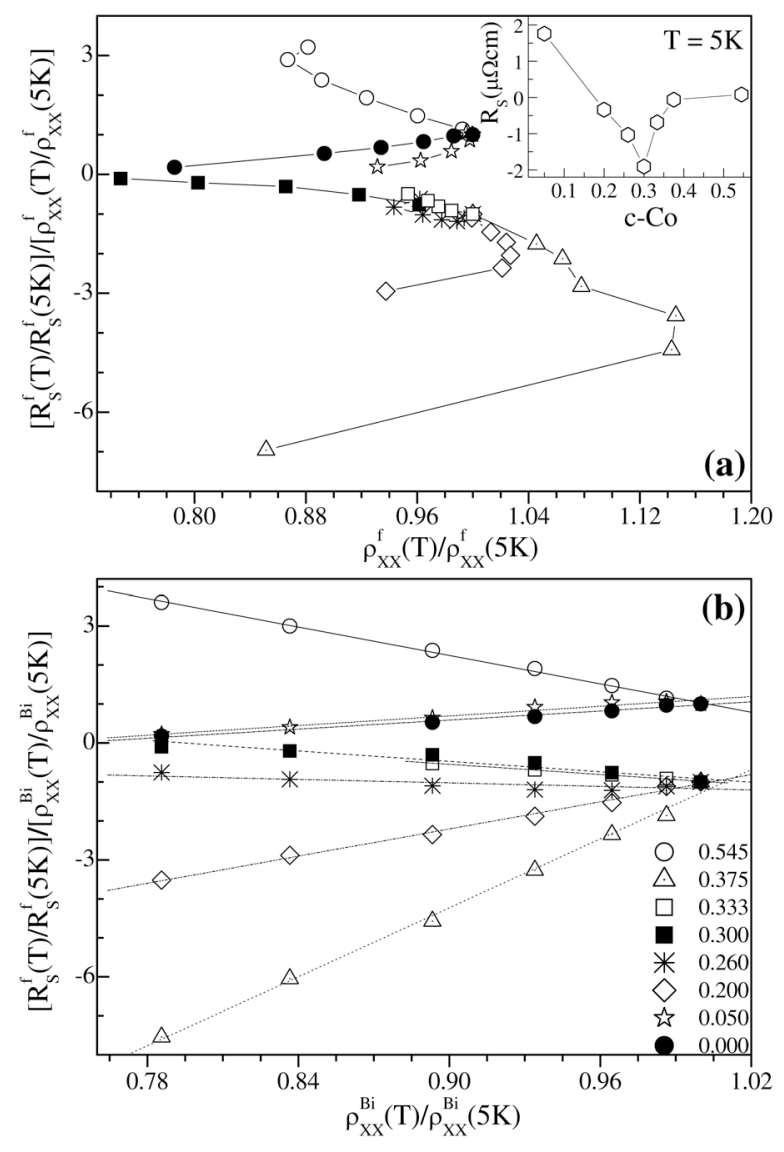

Fig. 6. (a) Normalized AHE coefficients $R_{S}(T)$ to $R_{S}(5 K)$ of the films are divided by the normalized film resistance at zero field $\rho_{x x}(T) / \rho_{x x}(5 K)$, and plotted as a function of $\rho_{x x}(T) / \rho_{x x}(5 K)$ of the film. Lines are guides to the eye. The inset shows $R_{S}$ values at $5 \mathrm{~K}$ as a function of c-Co. (b) The same $R_{S}(T) / R_{S}(5 K)$ values divided by the normalized resistance of $\mathrm{Bi}(15 \mathrm{~nm})$ film at zero field, as a function of the temperature dependence of the normalized resistance of $\mathrm{Bi}(15 \mathrm{~nm})$ film. Lines are linear fits satisfying Eq.2. Different symbols correspond to c-Co in different films and are the same in both plots.

\section{Conclusion}

In summary, magneto-transport measurements in polycrystalline $\mathrm{Co} / \mathrm{Bi}$ layered thin film structures reveal that $R_{H}^{f}$ or $\mathrm{R}_{0}$ values exhibit (Fig.4) a critical scaling close to a percolation threshold $\mathrm{p}_{\mathrm{c}}=0.3$, with a critical exponent $\mathrm{g}=0.3$. It was observed that between $0.2<\mathrm{c}<0.375$ : (i) both, the $\mathrm{OH}$ coefficient $\mathrm{R}_{0}$ and $R_{H}^{f}$ values become negative, evidencing a transition from $\mathrm{p}$ type to n-type majority charge carriers in this region, and (ii) a similar behaviour appears in the plot (Fig.6a, inset) of $\mathrm{R}_{\mathrm{S}}$ values as a function of c-Co at $5 \mathrm{~K}$. However, the sign of $R_{S}$ depends on the degree of orientation of [00l] crystallographic directions in $\mathrm{Bi}$ layers relative to film vertical direction, and is not related with Co concentration. It was shown that the obtained $\mathrm{R}_{\mathrm{S}}$ values satisfy Eq. 2 only if $\rho_{x x}^{f}(T)$ is replaced by $\rho_{x x}^{B i}(T)$ values observed in pure $\operatorname{Bi}(15 \mathrm{~nm})$ film. The last two results provide evidence that the dominant contributions to $\mathrm{AHE}$ are related with intrinsic and extrinsic mechanisms in $\mathrm{Bi}$ side of $\mathrm{Bi} / \mathrm{Co}$ interfaces.

\section{Acknowledgment}

This research has been co-financed by the European Union (European Social Fund - ESF) and Greek national funds through the Operational Program "Education and Lifelong Learning" of the National Strategic Reference Framework (NSRF) - Research Funding Program: Heracleitus II. Investing in knowledge society through the European Social Fund.

\section{References}

1. N. Nagaosa, J. Sinova, S. Onoda, A.H. MacDonald, N.P. Ong, Rev. Mod. Phys. 82, 1539 (2010)

2. X-J. Liu, X. Liu, J. Sinova, Phys. Rev. B 84, 165304 (2011)

3. K.-S. Wu, M.Y. Chern, J. Appl. Phys. 104, 033704 (2008)

4. Ph. Hofmann, Prog. Surf. Sci. 81, 191 (2006)

5. J. Lindemuth, B. Dodrill, IEEE Trans. Magn. 40, 2191 (2004)

6. D. Stroud and F. P. Pan, Phys. Rev. B 20, 455 (1979)

7. D. D. Franket and H.T. Chu, Phys. Rev. B 61, 13183 (2000).

8. C.M. Hurd, The Hall Effect in Metals and Alloys (Plenum, New York, 1972), Chap. 5.

9. D. Bergman, Y. Kantor, D. Stroud, I. Webman, Phys. Rev. Lett. 50, 1512 (1983).

10. J. Buxo, M. Saleh, G. Sarrabayrouse, G. Dorville, J. Berty, M. Brieu, Revue Phys. Appl. 15, 961 (1980)

11. D. Agassi and T.K. Chu, Apll. Phys. Lett. 51, 2227 (1987).

12. Y. Tian, L. Ye, X. Jin, Phys. Rev. Lett. 103, 087206 (2009). 